\title{
Rio + 20 : un sommet délétère ? Les désillusions de la conférence des Nations unies sur le développement durable des 20-22 juin 2012
}

Rio + 20: A Harmful Summit? Disillusionment with the United Nations Conference on Sustainable Development, June 20th - 22nd 2012

Philippe Ch.-A. Guillot

\section{OpenEdition \\ Journals}

Édition électronique

URL : http://journals.openedition.org/add/827

DOI : $10.4000 /$ add. 827

ISSN : 2606-1988

Éditeur

Presses universitaires de Rouen et du Havre

Édition imprimée

Date de publication : 1 avril 2015

Pagination : 137-175

ISSN : 1955-0855

Référence électronique

Philippe Ch.-A. Guillot, «Rio + 20 : un sommet délétère ? Les désillusions de la conférence des Nations unies sur le développement durable des 20-22 juin 2012 », Les Annales de droit [En ligne], 7 | 2013, mis en ligne le 03 mai 2018, consulté le 23 avril 2019. URL : http://journals.openedition.org/add/827 ; DOI $10.4000 /$ add 827 


\title{
Rio +20 : un sommet délétère ? Les désillusions de la conférence des Nations unies sur le développement durable des 20-22 juin 2012
}

\author{
Philippe Ch.-A. GuILLot
}

Vingt ans après la conférence des Nations unies sur l'environnement et le développement de Rio de Janeiro (alias Sommet de la terre, première conférence internationale au plus haut niveau de l'après guerre froide), s'est tenue dans la même ville, du 20 au 22 juin 2012, la conférence des Nations unies sur le développement durable (dite sommet Rio +20 ) qui s'est conclue par l'adoption par les représentants de 194 pays, dont 79 représentés par leur chef d'État ou de gouvernement, d'une déclaration - en fait, approuvée la veille de l'ouverture par les plénipotentiaires intitulée L'avenir que nous voulons ${ }^{1}$ et censée sauver la planète de la crise écologique majeure qui la menace, mais ce texte est généralement considéré comme n'étant pas à la hauteur des enjeux. L'égoïsme et la pusillanimité des États sont dénoncés comme responsables de cette déroute.

Il est vrai que d'aucuns n'attendaient rien du sommet, à l'instar du « contre-sommet des peuples», dont la déclaration fustige «le scénario voué à l'échec des fausses solutions défendues par les mêmes acteurs qui ont provoqué la crise mondiale» :

La dite «économie verte » est une des expressions de la phase financière actuelle du capitalisme qui utilise d'anciens et nouveaux mécanismes, comme l'augmentation de l'endettement public-privé, l'encouragement à la surconsommation, l'appropriation et la concentration de nouvelles technologies,

1. L'avenir que nous voulons, A/CONF. 216/L.1*. Le texte étudié dans le présent article est une traduction de courtoisie de l'Organisation des Nations unies (ONU) du texte original rédigé en anglais et n'est pas encore la traduction officielle. 
les marchés du carbone et de la biodiversité, la privatisation et l'aliénation des terres et les partenariats public-privé ${ }^{2}[\ldots]$

Cependant, nombreux sont ceux dont les sincères espoirs ont été amèrement dissipés. Pour l'ancienne ministre brésilienne de l'environnement Marina Silva, «les leaders » politiques ont choisi de ne pas avancer » : "Ils donnent la priorité à la crise économique et repoussent tout ce qui a trait à la protection de l'environnement ${ }^{3}$. " Dans des registres variés, mais quasi unanimement, les militants écologistes ${ }^{4}$ expriment leur profonde déception, à l'instar de Kumi Naidoo, directeur général de Greenpeace International, qui déclara le 22 juin 2012 : «On remet en ordre les fauteuils sur le pont du Titanic alors qu'il est en train de sombrer ${ }^{5}$. " Les organisations de défense des droits de l'homme sont également dépitées ${ }^{6}$. La presse n'est pas séduite non plus par les résultats du sommet :

La déception a surpassé l'ennui. [...] Dès l'ouverture du sommet, le rêve d'une meilleure protection de la biodiversité terrestre, d'une réglementation des océans, d'une réduction des grands déséquilibres mondiaux, d'une optimisation de l'efficacité énergétique, d’objectifs définis de développement durable pour toute la planète s'effondre ${ }^{7} \ldots$

Les journalistes Gilles van Kote et Thomas Wieder ne sont guère plus tendres pour cet «échec international » et citent en renfort le secrétaire général de l'ONU, pour qui les «efforts n'ont pas été à la mesure des défis qui nous attendent ${ }^{8}$ ». Et le président de la République française de renchérir: «Ces résultats, aussi appréciables soient-ils, sont en dessous

2. Déclaration finale du Sommet des peuples pour la justice sociale et environnementale, pour la défense des biens communs et contre la marchandisation de la vie, 22 juin 2012, disponible sur http://www.oree.org/gouvernance/rio+2o/html [consulté le 21 août 2012].

3. Libération, 23 juin 2012.

4. Pour un florilège, voir : Petit dictionnaire des réactions à Rio, http://www.ecobase21; net/Rioplusvingt/Rioplusvingt.html [consulté le 12 août 2012].

5. Cité dans http://www.levif.be/actualite/environnement/le-bilan_tres_ mitige-de-rio-20/article-4000120927459.htm [consulté le 26 juin 2012].

6. Voir par exemple l'avis de Human Rights Watch, http://www.hrw.org/fr/news/2012/ 06/22/rio-le-document-final-amoindri-par-les-opposants-aux-droits-humains. htm [consulté le 30 juin 2012].

7. Virginie Jacoberger-Lavoué, «Rio + 20, l'ordre sans progrès », http://www.valeursactuelles.com/actualit $\mid \% \mathrm{C}_{3} \backslash \% \mathrm{~A} 9 \mathrm{~s} /$ monde/rio- $20-1 \mid \% \mathrm{E}_{2} \backslash \% 8 \mathrm{o} \backslash$ $\% 990 r d r-s a n s-p r o g r \mid \% C_{3} \backslash \% \mathrm{~A} 8 \mathrm{c} 20120624 \cdot h t m l, 24$ juin 2012 [consulté le 5 juillet 2012].

8. Le Monde, 22 juin 2012. 
de nos responsabilités et de nos attentes ${ }^{9}$. » Il n'y a guère que le ministre burkinabè de l'environnement et du développement durable, Jean Koulidiati, pour considérer que : «La conférence de Rio +20 a été plutôt un succès en dépit de l'absence des chefs d'État de certaines grandes puissances mondiales comme les États-Unis d'Amérique, l'Allemagne, la Grande-Bretagne ${ }^{10}$; mais aussi au regard des résultats obtenus et des contraintes liées à des négociations multi-acteurs de ce type de rencontre, où plusieurs intérêts parfois divergents sont engagés ${ }^{11}$.»

En effet, les divergences entre États ont conduit à l'adoption d'un texte amphigourique mais manquant singulièrement de souffle et d'ambition. Le jargon technocratique, les truismes et les redondances peinent à masquer la vacuité de nombreuses dispositions qui, au mieux, ne font que rééditer d'anciennes promesses. L'inclusion de l' "économie verte » comme viatique du développement durable s'est faite au prix d'une dénaturation de cette nouvelle notion et la gouvernance environnementale, qui aurait dû être relancée, marque le pas. Les États se recroquevillent. Aucun traité nouveau n'a été signé, aucune négociation nouvelle n'a été ouverte. Il n'y eut même pas de prise de conscience planétaire, puisque la conférence donna lieu à un affrontement de souverainetés. Les quelques nouveaux engagements politiques contenus dans la déclaration ne sont assortis d'aucun plan de financement précis, ni d'objectifs chiffrés. Le désappointement est évidemment à la mesure des espoirs imprudemment mis en cette conférence commémorative, qui semble plutôt célébrer l'enterrement du développement durable, si ce n'est en tant que panacée, du moins en tant que mythe sorélien.

Du haut de ses vingt ans d'âge, le concept de développement durable aurait donc perdu sa valeur mobilisatrice et serait réduit à une simple incantation de convenance. Une étude à tête reposée de la déclaration finale $\mathrm{du}$ sommet Rio +20 dément cet avis de décès prématuré du développement durable. Certes, il n'a plus la fraîcheur d'il y a vingt ans et la conférence ne fut pas pour lui une cure de jouvence, mais en dépit des oppositions plus nombreuses et plus vindicatives provenant de certains États et d'une part importante de la «société civile transnationale», il demeure une valeur internationale consensuelle. Ce consensus est sans

9. Voir son discours de Rio, http://www.elysee.fr/president/les-actualites/discours/ 2012/discours-de-m-le-president-de-la-republique.13465.html [consulté le 24 juin 2012].

10. Et la Russie...

11. Cité par Germaine Kere et Djakaridja Savadogo, "Bilan burkinabè de Rio + 20 ", Le Pays, $\mathrm{n}^{\circ}$ 5170, 7 août 2012, http://www.lepays.bf/IMG/article_PDF/article_a6872 [consulté le 25 août 2012]. 
doute possible car chaque État ou "partie prenante» (Stakeholder) en a sa propre compréhension. Puisque justement le développement durable est alors « une notion passe-partout, qu'il faut invoquer rituellement pour justifier n'importe quelle proposition, mais qui revêt des significations très variées ${ }^{12}$ ", il convient de rappeler comment ladite notion est apparue, puis fut consacrée il y a vingt ans avant de se pencher sur l'approche empruntée lors du sommet Rio +20 du concept qui désormais intègre l'évanescente "économie verte». Si les promoteurs de cette "économie verte" ne peuvent qu'être désenchantés par la version édulcorée retenue par la déclaration, les principales désillusions sont surtout afférentes au délitement de la gouvernance internationale environnementale ainsi qu'à certaines omissions de la déclaration.

\section{Le développement durable, mythe du $x^{\mathrm{e}}{ }^{\mathrm{e}}$ siècle}

Inventée en 1974 par Henry Kissinger pour contrer les diatribes anti-développementalistes des écologistes, mais récupérée et recyclée par l'Union internationale pour la conservation de la nature ${ }^{13}$ (UICN) en 1980, la notion de développement durable (Sustainable Development) fut popularisée - d'abord sous l'anglicisme "développement soutenable» - par la commission de l'ONU présidée par Gro Harlem Brundtland, qui la définissait ainsi : "Un développement qui répond aux besoins du présent sans compromettre la capacité des générations futures à répondre aux leurs ${ }^{14}$. "Se fondant sur les travaux de cette commission, José A. Prades la glose en une vision des grands principes éthiques devant orienter le devoir et l'idéal de responsabilité dans la gestion humaine (Stewardship) de la planète, à savoir l'harmonisation aux niveaux local, régional et mondial - des exigences de la sauvegarde de l'environnement avec celles du progrès économique et social. Le

12. François Ascher, La République contre la ville. Essai sur l'avenir de la France urbaine, Paris, Éditions de l'aube, 1998, p. 74.

13. «[...] si l'objectif du développement est la prospérité économique et sociale, celui de la conservation est le maintien de la capacité de la terre d'assurer aussi bien le développement durable de l'humanité que la pérennité de toute vie », UICN, Stratégie mondiale de la conservation de la nature, 1980, cité dans Jean-Claude Pierre, Pourvu que ça dure! Le développement durable en question, Le Faouët, Liv'Éditions, 2006, p. 101-102 ; sur cette structure associative faisant cohabiter représentants des gouvernements et associations et personnalités indépendantes, voir : Roland Séroussi, Droit international de l'environnement, Paris, Dunod, 2012, p. 167-169 et le site: http://www.uicn.org.

14. Commission mondiale sur l'environnement et le développement, Notre avenir à tous, Montréal, Les Éditions du fleuve, 1987, p. 51. 
développement durable s'analyse comme « un processus de transformation dans lequel l'exploitation des ressources, la direction des investissements, l'orientation des techniques et le changement des institutions se font de façon harmonieuse et renforcent le potentiel présent et à venir qui permet de mieux répondre aux besoins et aux aspirations de l'humanité ». Ces changements sont déterminés en fonction des besoins de l'humanité, des limites de l'environnement et de l'accès équitable de tous aux différentes ressources:

Il y a développement réel lorsque le processus de croissance conduit à un état d'harmonie entre les êtres humains et le patrimoine naturel de la planète; [...] la survie de l'humanité et son bien-être peuvent dépendre de la façon dont on saura élever le développement soutenable au rang d'une éthique mondiale ${ }^{15}$.

Cette notion s'est progressivement imposée comme le thème mobilisateur par excellence du $\mathrm{xxI}^{\mathrm{e}}$ siècle. Il sied alors de retracer comment elle est devenue un leitmotiv du droit international de l'environnement et d'indiquer quelle substance ce dernier lui donne, avant d'examiner les contours du concept dessinés à Rio, vingt ans plus tard.

\subsection{Le développement durable de Rio 1992 à nos jours}

À Rio de Janeiro en 1992, le développement durable apparaît dans la déclaration finale de la conférence des Nations unies sur l'environnement et le développement qui contient 27 principes $^{16}$ et est complétée par le plan d'action pour le xxi siècle (Agenda 21), lequel comprend des centaines de recommandations que les États et les institutions internationales doivent mettre en ouvre pour atteindre certains objectifs (réduction du gaspillage des ressources naturelles; lutte contre la pauvreté; protection de l'atmosphère, des océans, de la faune et de la flore, etc.) avant l'an 2000 ; les collectivités infra-étatiques sont invitées à mettre en place des «agendas 21 » locaux. Le concept traduit d'abord la volonté onusienne d'élaborer une nouvelle idéologie consensuelle dans l'après guerre froide. Aussi repose-t-il sur des principes (intégration, coopération, équité et participation) qui sont d'autant plus facilement acceptés qu'ils laissent une large place à l'interprétation, mais il n'est pas un principe juridique.

15. José A. Prades, L'Éthique de l'environnement et du développement, Paris, PUF, «Que sais-je?», 1995, p. 40-41.

16. Doc. ONU A/CONF.151/26 (vol. I), annexe I (1992). Voir aussi Laurence Boisson de Chazournes, Richard Desgagné, Makane M. Mbengue et Cesare Romano, Protection internationale de l'environnement, Paris, Pédone, 2 éd., 2005, p. 13-47. 


\subsubsection{L'idéologie onusienne}

L'activité de l'ONU a d'abord consisté à rechercher les moyens de réunir les États membres autour d'un slogan consensuel, avant de définir des objectifs et des normes. À cet égard, le terme de «développement durable » apparaît comme un compromis entre la logique développementaliste de la Charte des Nations unies et la nouvelle demande sociale, émanant principalement des opinions publiques du Nord, pour une protection de l'environnement. Le terme « environnement» ne figurant pas dans la Charte, contrairement à celui de « développement», il était donc nécessaire pour fonder une compétence environnementale de l'ONU de s'appuyer sur ce dernier concept, lequel est bien entendu un objectif apprécié des États du Sud. Au sortir de la guerre froide, il s’agissait aussi pour l'ONU d'éviter qu'au conflit Est-Ouest enfin enterré ne se substitue un affrontement Nord-Sud. Néanmoins, le clivage entre un hémisphère nord soucieux de la protection de l'environnement qu'il a puissamment dégradé au fil des siècles et un hémisphère sud qui considérerait cette protection de l'environnement comme un luxe doit être relativisé. D'une part, tous les États du Nord ne sont pas favorables à un droit international de l'environnement trop directif - les États-Unis d'Amérique ont une législation interne souvent exemplaire en matière de protection de l'environnement, mais sont très réticents à soutenir l'extension des compétences internationales en ce domaine ${ }^{17}$ - d'autre part, les pays du Sud ont plus à gagner qu'à perdre au développement d'un droit international de l'environnement. En effet, selon le programme des Nations unies pour le développement (PNUD), «la dégradation de l'environnement frappe presque toujours ceux qui vivent dans la pauvreté ${ }^{18} »$. On en vient ainsi, à envisager la protection de l'environnement comme une composante du développement humain durable :

L'environnement durable est un élément majeur du développement humain durable et fait partie à ce titre des priorités du PNUD dont la toute première est l'élimination de la pauvreté [...] car pour survivre, les plus pauvres détruisent dans un but de profit immédiat les ressources naturelles qui les

17. Voir Garry C. Bryner, "The United States: "Sorry-Not Our Problem" ", dans William M. Lafferty et James Meadowcroft (dir.), Implementing Sustainable Development. Strategies and Initiatives in High Consumption Societies, Oxford University Press, 2000, p. 273-301; néanmoins, l'administration Obama est un peu plus favorable à une approche multilatérale du développement durable : voir Jean-Michel Valentin, «Rio, miroir de la nouvelle pensée stratégique américaine? », Diplomatie, $\mathrm{n}^{\mathrm{o}} 58,2012, \mathrm{p} \cdot 36-37$.

18. PNUD, Rapport sur le développement humain, Paris, Économica, 1998, p. 74. 
environnent, coupant par là même les vivres aux générations suivantes, et perpétuant le cercle infernal de la pauvreté ${ }^{19}$.

L'administrateur du PNUD, James Gustave Speth, complète :

Un développement humain durable n'a pas pour objectif premier de créer la croissance, mais de distribuer équitablement ses bénéfices ; il vise à régénérer l'environnement au lieu de le détruire; il permet aux populations d'accéder à l'autonomie au lieu de les marginaliser; il élargit leurs choix et leurs possibilités, tout en permettant aux personnes de participer aux décisions qui les concernent. Un développement humain durable donne la priorité aux pauvres, à la préservation des milieux, à l'emploi et aux femmes ${ }^{20}$.

La priorité est donc donnée à la satisfaction des besoins fondamentaux, en mettant l'accent sur la sécurité alimentaire et une agriculture durable, sur les ressources en eau potable et l'environnement aquatique, sur une utilisation viable de l'énergie, particulièrement via les sources d'énergie renouvelables et la conservation de l'énergie, et sur la bonne gestion des ressources forestières.

Reposant sur une vision rationnelle et optimiste de l'avenir selon laquelle le progrès technique et l'action humaine pourraient relever les défis écologiques, le développement durable est incontestablement un concept libéral qui peut être considéré comme "[l']expression d'une nouvelle "idéologie internationale" consistant à étendre les règles du commerce international aux biens environnementaux qui y avaient jusque là échappé au nom du principe de la non-exploitabilité du vivant » : «À cet égard, le développement durable apparaît comme l'expression d'un phénomène de "planétarisation" du droit (A. Kiss) ou si l'on veut, comme le signe ultime d'une évolution vers sa globalisation $^{21}$.» Cette mondialisation du droit fut parrainée par les chefs d'État ou de gouvernement de la plupart des pays (dont ceux des plus puissants) à la conférence de Rio en 1992.

19. Bertrand Coppens, «Le rôle du PNUD dans la promotion d'un développement humain durable », dans Sandrine Maljean-Dubois et Rostane Mehdi (dir.), Les Nations unies et la protection de l'environnement: la promotion d'un développement durable, Paris, Pédone, 1999, p. 51-52; voir aussi Fatma-Zohra Ksentini, «Human Rights, Environment and Development ", dans UNEP's New Way Forward: Environmental and Sustainable Development, Nairobi, PNUE, 1995, p. 95-114.

20. Cité par Bertrand Coppens, art. cité, p. 52.

21. Stéphane Doumbé-Billé, "Les mécanismes de suivi de la mise en œuvre du développement durable", dans S. Maljean-Dubois et R. Mehdi (dir.), op. cit., p. 105-106. 


\subsubsection{Le développement durable selon la déclaration de Rio de 1992}

À l'instigation du Brésil, il y eut des tentatives lors de la conférence de Rio de 1992 pour supplanter l'expression «droit international de l'environnement " par celle de «droit international du développement durable», mais elles n'aboutirent pas, même si le droit international de l'environnement est désormais «mâtiné de droit international du développement ${ }^{22}$ ", puisque les «États industrialisés ont dû comprendre que l'on ne peut pas protéger l'environnement de la planète contre ou même sans la très large majorité de la population mondiale qui, tout en étant pauvre, détient aussi une forte proportion de certaines ressources naturelles ${ }^{23} »$. La déclaration de Rio de 1992 ne définit pas, à proprement parler, le développement durable mais se structure autour de quatre principes phares : intégration, coopération, équité et participation.

\subsubsection{Le principe d'intégration}

Il consiste à se départir des approches sectorielles - lutte contre certaines pratiques génératrices de pollution, protection de certaines espèces animales ou végétales, protection de l'environnement dans des régions géographiques déterminées - au profit d'une approche globale, dans tous les sens du terme, car « la protection de l'environnement doit faire partie intégrante du processus de développement et ne peut être considérée isolément» (déclaration de Rio, principe 14). Si cette rédaction présente une apparente clarté, on a pu écrire à son sujet: « De tous les principes généraux du droit de l'environnement, le principe d'intégration est sans doute le plus ambigu, celui qui offre le plus grand nombre de lectures, le terrain sur lequel l'argumentation juridique dissimule le plus mal les affrontements idéologiques ${ }^{24}$.» En effet, selon les priorités que s'assignera un État, la protection de l'environnement pourra être vue soit comme subordonnée au développement économique, soit comme, au contraire, s'imposant à celui-ci.

22. Sandrine Maljean-Dubois et Rostane Mehdi, «Environnement et développement, les Nations unies à la recherche d'un nouveau paradigme», dans S. Maljean-Dubois et R. Mehdi (dir.), op. cit., p. 24.

23. Alexandre Kiss et Jean-Pierre Beurier, Droit international de l'environnement, Paris, Pédone, 4 éd., 2010, p. 52.

24. Jean-François Neuray, Droit de l'environnement, Bruxelles, université libre de Bruxelles - Bruylant, 2001, p. 89. 


\subsubsection{Le principe de coopération}

Au-delà de la simple obligation de bon voisinage, ce principe tend vers la solidarité internationale et a pour conséquence pratique d'obliger les États à s'informer mutuellement et à se consulter sur les activités ou événements susceptibles d'avoir des effets transfrontières sur l'environnement (principe 19). Il faut donc que les États se fassent confiance et collaborent de bonne foi, ce qui s'apparente souvent à un vœu pieux, d'autant plus qu'un État avouant être à l'origine d'une pollution transfrontière engagera sa responsabilité internationale.

\subsubsection{Le principe d'équité}

Ce principe se décline en une équité intragénérationnelle consistant en la prise en compte des coûts sociaux injustifiés que pourrait entraîner l'adoption de mesures pour la protection de l'environnement par les pays en développement, en particulier les pays les moins avancés (principe 6) et qui débouche par l'affirmation de «responsabilités communes mais différenciées ${ }^{25}$ " (principe 7), et en une équité intergénérationnelle résidant en la protection des «droits des générations futures » - euphémisme pour devoirs des générations présentes, que l'on légitime par le proverbe africain cité par Antoine de Saint-Exupéry dans Terre des hommes : « La terre ne nous appartient pas, nous l'empruntons à nos enfants »-, fondée sur l'utilisation rationnelle des ressources naturelles renouvelables et sur les principes de précaution (principe 15), pollueur-payeur (principe 16) et de prévention (principe 17).

Si l'équité intragénérationnelle se traduit par des transferts financiers, voire des transferts de technologie, Nord-Sud dans de nombreux traités, elle peut aussi être source de craintes pour la préservation de l'environnement :

Le principe de responsabilité commune mais différenciée est l'expression d'une revendication des États en retard de développement suivant laquelle ils auraient le droit de porter autant atteinte à l'environnement que ne l'ont fait, avant eux, les pays industrialisés. Il reste que le principe pourrait tout aussi bien conduire le second groupe, en vertu de la responsabilité accrue qui lui incombe, à cesser d'acheter des produits du Tiers-Monde exploités sans respect pour l'environnement, notamment les bois tropicaux ${ }^{26}$.

25. Voir Mohammed Abdelwahab Bekhechi, «Le droit international à l'épreuve du développement durable », Annuaire de La Haye de droit international, vol. 6, 1993, p. $70-72$.

26. Jean-François Neuray, op. cit., p. 72. 


\subsubsection{Le principe de participation}

Ce principe vise à favoriser, au niveau local, l'association du public ou de la société civile aux décisions dans les matières relatives au développement socio-économique ou à la protection de l'environnement. Il ne s'agit pas d'une simple pétition en faveur de la démocratie, mais de la constatation que les régimes totalitaires ou autoritaires respectent moins l'environnement que les sociétés démocratiques. A contrario, les deux révolutions démocratiques en Union soviétique et en Hongrie en 1989-1991 peuvent être directement reliées aux conséquences écologiques de l'accident de Tchernobyl et de la polémique au sujet du barrage de Nagymaros. Au demeurant, dans la "plus grande démocratie du monde ", depuis la catastrophe de Bophal, les juridictions indiennes mettent plus fortement l'accent sur les Droits de l'homme à l'occasion de contentieux environnementaux ${ }^{27}$. Encore faut-il cependant que les États laissent leur population s'exprimer...

\subsubsection{L'absence de juridicité d'un concept irriguant les politiques publiques et les activités privées}

Issu de la déclaration de Rio de 1992, le développement durable, bien que de plus en plus réceptionné dans des traités, est surtout présent dans des documents de droit programmatoire (soft law) et manque donc de force obligatoire, mais, même "si l'Action 21 ne constitue effectivement qu'un programme dénué en principe de force contraignante, nul ne se risquerait à suggérer que la Commission du développement durable (CDD) peut en ignorer l'existence ${ }^{28}$ ".

Si le concept de développement durable a incontestablement une valeur politique, jusqu'à présent la jurisprudence internationale ne l'a pas élevé au rang de principe juridique contrairement aux principes juridiques sur lesquels il s'appuie ${ }^{29}$. En effet, dans son arrêt rendu le 25 septembre 1997 relatif au différend opposant la Hongrie et la

27. Voir Alan Boyle, «Economic Growth and Protection of the Environment: The Impact of International Law and Policy", dans id., Environmental Regulation and Economic Growth, Oxford, Clarendon Press, 1994, p. 178.

28. Sandrine Maljean-Dubois et Rostane Mehdi (dir.), op. cit., p. 17. Sur le programme action 21, voir Laurent Lucchini, «Le contenu et la portée de l'Agenda 21 », ibid., p. 69-77.

29. Voir Nico Schrijver, The Evolution of Sustainable Development in International Law: Inception, Meaning and Status, La Haye, Académie de droit international, 2008, p. 141-150 [reprise de la leçon publiée au Recueil des cours, vol. 329, 2007, p. 217-412]. 
Slovaquie à propos du projet de barrage Gabčikovo-Nagymaros, la Cour internationale de justice a simplement qualifié de «concept» le développement durable ${ }^{30}$. L'attendu pertinent mérite d'être cité :

La Cour ne perd pas de vue, dans le domaine de la protection de l'environnement, que la vigilance et la prévention s'imposent en raison du caractère souvent irréversible des dommages causés à l'environnement et des limites inhérentes au mécanisme même des réparations de ce type de dommage. Au cours des âges l'homme n'a cessé d'intervenir dans la nature pour des raisons économiques et autres. Dans le passé, il l'a souvent fait sans tenir compte des effets sur l'environnement. Grâce aux nouvelles perspectives qu'offre la science et à une conscience croissante des risques que la poursuite de ces interventions à un rythme inconsidéré et soutenu que représenterait pour l'humanité, et qu'il s'agisse des générations actuelles ou futures, de nouvelles normes et exigences ont été mises au point, qui ont été énoncées dans un grand nombre d'instruments au cours des deux dernières décennies. Ces normes nouvelles doivent être prises en considération et ces exigences nouvelles convenablement appréciées, non seulement lorsque des États envisagent de nouvelles activités, mais aussi lorsqu'ils poursuivent des activités qu'ils ont engagées dans le passé. Le concept de "développement durable » traduit bien cette nécessité de concilier développement économique et protection de l'environnement.

Le concept de développement durable est donc un fil conducteur mais non un principe général du droit international. Ce qui ne l'empêche pas d'influer non seulement sur les traités internationaux et l'action des organisations internationales, mais aussi sur le droit interne des États puisque sa finalité est d'être appliqué au niveau national, voire infra-étatique. Concrètement, le concept de développement durable se traduit notamment par l'incorporation dans les procédures de décision publique d'une dimension environnementale: les considérations liées à la protection de l'environnement sont de plus en plus prises en compte dans les planifications et les outils de gestion publics, traduisant ainsi un «verdissement» (greening) des politiques publiques. À ce processus d'assimilation des préoccupations environnementales aux éléments à prendre en considération dans les décisions publiques s'ajoute la tendance à l'intégration d'une gestion environnementale dans les polices administratives afin d'adopter une méthode moins parcellaire de la protection de l'environnement en général et de la lutte contre les nuisances en particulier ${ }^{31}$. De plus en plus, ce concept s'étend au secteur privé :

30. $\$ 140$, Recueil des arrêts et avis de la Cour, 1997, p. 77.

31. Tim Jewell, "Public Law and the Environment: The Prospects for DecisionMaking», dans Tim Jewell et Jenny Steele, Law in Environmental Decision-Making, Oxford, Clarendon Press, 1998, p. 79-81. 
Le développement durable est aujourd'hui polymorphe et transversal, il est non seulement une culture (née dans les cercles multilatéraux OutreAtlantique) mais également un système de gouvernance. Ses déclinaisons sont économiques et financières (la transparence, un sous-concept du développement durable); techniques (sécurité, précaution, durabilité), sociales (égalité des droits humains, non-discrimination), juridiques (la bonne gouvernance d'entreprise), comportementales (l'entreprise citoyenne). Pour l'entreprise, tous ces impératifs ont été regroupés sous le nom de responsabilité sociale $^{32}$, pendant privé du développement durable ${ }^{33}$.

En effet, par conviction ou par intérêt bien compris, les entreprises intègrent des aspects environnementaux et sociaux dans leurs chaînes de production ou dans leur management et entrent dans des procédures de certification telles qu'ISO $14001^{34}$ ou autres démarches volontaires sectorielles ${ }^{35}$; d'autres entreprises, moins vertueuses, ne font du développement durable qu'un simple argument mercatique et se contentent de « repeindre en vert» (greenwashing) leurs produits ou leur publicité, ce qui témoigne néanmoins du souci chez les consommateurs du développement durable.

\subsection{Le développement durable, vingt ans après}

Selon l'Institut du développement durable et des relations internationales (IDDRI) de Sciences Po Paris, L'Avenir que nous voulons est un « texte sans vision d'ensemble et accumulant des points et sections spécifiques, et, qui dès les réunions préparatoires, a fait l'objet de divergences fortes ${ }^{36}$ ». Sans prétention à l'exhaustivité, il convient d'examiner les

32. Également appelée responsabilité sociétale.

33. Claude Revel, La Gouvernance mondiale a commencé. Acteurs, enjeux, influences... et demain?, Paris, Ellipses, 2006, p. 26.

34. Voir Matthew Potoski et Elizabeth Elwakeil, «International Organization for Standardization 14001 ", dans Thomas Hale et David Held (dir.), Handbook of Transnational Governance. Institutions and Innovations, Cambridge et Malden, Polity Press, 2011, p. 295-302.

35. Voir, pour le secteur bancaire, Christopher Wright, «The Equator Principles », ibid., p. 229-236; pour l'industrie minière, pétrolière et gazière, Haelmut Weidner, « Extractive Industries Transparency Initiative », ibid., p. 236-243; pour le commerce équitable, Kate McDonald, «The Fair Trade System », ibid., p. 252-259; pour la sylviculture, Philipp Pattberg, «Forest Stewardship Council», ibid., p. 265-273; pour la pêche, Thomas Hale, «Marine Stewardship Council», ibid., p. 308-314; et pour les sociétés transnationales, Elisa Morgera, «O.E.C.D. Guidelines for Multinational Enterprises », ibid., p. 314-322, et Thomas Hale, «United Nations Global Compact », ibid., p. 350-356.

36. Lettre de l'IDDRI, $\mathrm{n}^{\mathrm{o}} 31$, juillet 2012, http://www.iddri.org/Iddri/Lettre-de-1-Iddri/ Rio+20-et-apres.htm [consulté le 9 août 2012]. 
points les plus généraux par lesquels la déclaration de 2012 redéfinit les pourtours du développement durable et notamment la controversée notion d'« économie verte » qui ressort quelque peu vidée de sa substance.

\subsubsection{Le développement durable dans la déclaration de Rio +20}

La déclaration est un long texte de 283 paragraphes - soit 60 pages dans la version française - à la rédaction souvent indigeste, tant du fait du style diplomatico-technocratique que des très fréquents renvois, explicites ou implicites, à des instruments antérieurs, mais il est toutefois possible d'identifier, d'une part, une lettre, c'est-à-dire la confirmation d'engagements précédents et les novations de lege feranda et, d'autre part, un esprit guidant les politiques publiques appelées à être mises en œuvre.

\subsubsection{La lettre: continuité et innovations}

La déclaration de Rio +20 n'est évidemment pas apparue ex nihilo, au contraire elle revendique une filiation avec d'autres instruments dont elle reprend les principes leur conférant ainsi un caractère coutumier, mais, contrairement à ce que laissent entendre des commentaires prématurés, elle comprend de non négligeables nouveautés.

\section{Le couronnement des normes juridiques du développement durable}

En célébrant le vingtième anniversaire du Sommet de la terre, les délégations ne souhaitaient évidemment pas marquer une rupture, mais, au contraire, s'inscrire dans la continuité. Sans surprise, L'avenir que nous voulons entend bâtir sur les fondations posées depuis plusieurs décennies avec la réaffirmation de la déclaration de Stockholm du 16 juin 1972, de la déclaration de Rio de 1992, du plan de mise en œuvre de Johannesburg de 2002, du programme d'action de La Barbade et de la stratégie de Maurice pour le développement durable des petits États insulaires, du programme d'Istanbul en faveur des pays les moins avancés, du programme d'action d'Almaty pour les États sans littoral et de transit, de la déclaration politique sur les besoins de développement de l'Afrique et du nouveau partenariat pour le développement de l'Afrique (NEPAD), de la déclaration du millénaire, du document final du sommet mondial de 2005, du consensus de Monterrey et de la déclaration de Doha sur le financement du développement, du document final de la réunion plénière de haut niveau l'assemblée générale de l'ONU sur 
les objectifs du millénaire pour le développement ${ }^{37}$, du programme d'action sur la population et le développement et de la déclaration et du programme d'action de Beijing ${ }^{38}$.

La confirmation de ces grands textes internationaux du développement durable et les références à certains traités associés ${ }^{39}$ érigent les principes juridiques qu'ils contiennent en principes du droit international général, la déclaration traduisant incontestablement une opinio juris sur leur force obligatoire et universelle. C'est pourquoi, la déclaration des juristes de l'environnement sur les engagements post-Rio +20 prend acte qu'ainsi le texte "consacre les acquis environnementaux de Rio 1992, en leur donnant un caractère coutumier au sens du droit international, empêchant désormais toute régression ${ }^{40} "-$ la reconnaissance de la "non régression» comme principe du droit de l'environnement est l'actuel cheval de bataille du doyen Michel Prieur, président du Centre international de droit comparé de l'environnement ${ }^{41}$.

37. Doc. ONU A/REs/55/2, 8 septembre 2000, Voir aussi Laurence Boisson de Chazournes, Richard Desgagné, Makane M. Mbengue et Cesare Romano, op. cit., p. 17-19.

38. Voir L'avenir que nous voulons, op. cit., $\$ 14-17$.

39. Le $\$ 192$ exhorte « les parties à la convention-cadre des Nations unies sur les changements climatiques et au protocole de Kyoto à respecter intégralement leurs engagements ainsi que les décisions adoptées dans le cadre de ces instruments [...] y compris lors de la dix-septième conférence des parties à la convention-cadre des Nations unies sur les changements climatiques et de la septième conférence des parties agissant comme réunion des parties au protocole de Kyoto tenue à Durban (Afrique du Sud) du 28 novembre au 9 décembre 2011 »; le $\$ 199$ prend «note de l'adoption du protocole de Nagoya sur l'accès aux ressources génétiques et le partage juste et équitable des avantages découlant de leur utilisation et [invite] les parties à la convention sur la diversité biologique à ratifier ou à accéder à ce protocole afin de garantir son entrée en vigueur dès que possible»; le $\$ 207$ affiche la détermination des signataires «à soutenir et à renforcer la mise en œuvre de la convention [des Nations unies sur la lutte contre la désertification] et de son plancadre stratégique décennal (2008-2018), y compris en mobilisant des ressources financières suffisantes et prévisible en temps voulu».

40. Déclaration adoptée à Rio de Janeiro, le 17 juin 2012, à l'initiative du Centre international de droit comparé de l'environnement, de la fondation G. Fargas et de l'Environmental Law Institute, http://www.cidce.org/rio/pdfl\%2oappel/ Déclaration $\mid \% 20$ post $\mid \% 20$ Rio.pdf [consulté le 15 juillet 2012].

41. Voir Michel Prieur, "De l'urgente nécessité de reconnaitre le principe de "non régression" en droit de l'environnement ", Revue roumaine de droit de l'environnement, vol. 8, $\mathrm{n}^{\mathrm{o}} 2$, 2010, p. 9-29. 
L'acceptation d'un héritage n'est pas antithétique de la volonté de le faire fructifier. La déclaration de Rio +20 est plus audacieuse que ces devancières, puisque les États présents ont expressément reconnu "que la démocratie, la bonne gouvernance et l'état [sic] de droit, au niveau national et au niveau international, ainsi qu'un environnement favorable, sont des conditions sine qua non du développement durable, notamment d'une croissance économique durable et profitant à tous, du développement social, de la protection de l'environnement et de l'élimination de la faim et de la pauvreté ${ }^{42}$. " Les cyniques y verront un hommage du vice à la vertu, compte tenu du fait que la majorité des États, notamment dans les pays en développement, ne sont guère respectueux de ces principes. Il est vrai que le mot "démocratie » est polysémique et que la plupart des régimes s'affirment démocratiques, tandis que la «bonne gouvernance» peut donner lieu à de multiples interprétations ${ }^{43}$. Cependant, comme le paragraphe précédent réaffirme l'importance de la Déclaration universelle des droits de l'homme et souligne que tous les États sont tenus par la Charte de l'ONU de respecter les Droits de l'homme et les libertés fondamentales, les termes "démocratie » et "gouvernance» se comprennent en un sens libéral. Si la déclaration se réfère aux Droits de l'homme, elle n'en proclame pas de nouveau, au dépit des organisations non gouvernementales (ONG) de protection des Droits de l'homme qui déplorent que le «Saint-Siège [ait] mené l'attaque contre les droits reproductifs avec le soutien du $\mathrm{G} 77^{44}$ », ce qui signifie donc que la majorité des États, représentant la majorité de la population mondiale, ne désirent pas les reconnaître...

42. L'avenir que nous voulons, op. cit., $\$ 10$.

43. Voir Philippe Moreau Defarges, La Gouvernance, Paris, PUF, «Que sais-je? ", 2003, p. 38-44. Pour une vision onusienne officieuse, voir PNUD, Reconceptualising Governance, Discussion Paper, $\mathrm{n}^{\circ}$ 2, 1997, 93 p. Plus généralement sur la gouvernance au niveau interne, voir Jean-François Kerléo, «La gouvernance en droit. À la poursuite de l'imaginaire juridique de la notion ", Les Annales de droit, $\mathrm{n}^{\circ}$, 2011, p. 83-123; Arnaud Cabanes, Essai sur la gouvernance publique. Un constat sans concession, quelques solutions sans idéologie, Paris, Gualino, 2004; Jean-Pierre Gaudin, Pourquoi la gouvernance?, Paris, Presses de Sciences Po, 2002. Pour une critique, voir Madeleine Arondel-Rohaut et Philippe Arondel, Gouvernance: une démocratie sans le peuple?, Paris, Ellipses, 2007 ; Pierre Calame, avec la collaboration de Jean Freyss et Valéry Garandeau, La Démocratie en miettes: pour une révolution de la gouvernance, Paris, Descartes et $\mathrm{C}^{\mathrm{ie}}, 2003$.

44. Human Rights Watch, http://www.hrw.org/fr/news/2012/06/22/ rio-le-document-final-amoindri-par-les-opposants-aux-droits-humains.htm [consulté le 30 juin 2012]. 
En revanche, le droit à la santé connaît une avancée puisque, pour la première fois à un tel niveau, les États s'engagent "à renforcer les systèmes de santé en vue d'assurer une couverture universelle équitable et de promouvoir l'accès à un coût abordable à des services de prévention, de traitement, de soins et de soutien psychosocial pour les maladies non transmissibles, en particulier les cancers, les maladies cardiovasculaires, les maladies respiratoires chroniques et le diabète $\mathrm{e}^{45}$ ». C'est pourquoi le président François Hollande a pu déclarer: "Le développement durable n'est pas une contrainte. C'est un levier. Ce n'est pas non plus un outil de protection pour les plus favorisés contre les autres mais de promotion d'une conception de l'humanitét ${ }^{46}$.»

\subsubsection{L'esprit: une conception solidaire de l'humanité}

Cette conception de l'humanité repose sur la solidarité et l'accent est mis sur l'importance de l'éducation et de la participation.

\section{Le développement durable fondé sur la solidarité}

Digne héritière des grands textes du développement durable qui l'ont précédé, la déclaration affirme:

[...] il importe d'aider les pays en développement à éliminer la pauvreté et à favoriser l'autonomisation des pauvres et des personnes en situation vulnérable, notamment en éliminant les obstacles auxquels ils sont confrontés et en renforçant les capacités de production, en développant l'agriculture durable et en favorisant le plein emploi productif et un travail décent pour tous, complétés par des politiques sociales efficaces, y compris la mise en place de filets de protection, le but étant d'atteindre les objectifs de développement convenus au plan international, y compris les objectifs du millénaire pour le développement ${ }^{47}$.

De prime abord, le développement durable semble universaliser le modèle de l'État social, alors même que la mondialisation le remet en

45. L'avenir que nous voulons, op. cit., $\$ 141$.

46. Voir son discours de Rio, http://www.elysee.fr/president/les-actualites/discours/ 2012/discours-de-m-le-president-de-la-republique.13465.html [consulté le 24 juin 2012].

47. L'avenir que nous voulons, op. cit., $\$ 23$; le $\$ 247$ précise que «les objectifs de développement durable doivent être concrets, concis et faciles à comprendre, en nombre limité, ambitieux, d'envergure mondiales et susceptibles d'être appliqués dans tous les pays compte tenu des réalités, des ressources et du niveau de développement respectifs de ceux-ci ainsi que des politiques et des priorités nationales. » 
cause $^{48}$; une analyse plus poussée, s'appuyant notamment sur les travaux relatifs à l'« économie verte» ou à la "croissance verte", amène à penser qu'il ne s'agit pas nécessairement d'une résurrection de l'Étatprovidence qui mondialiserait la social-démocratie - ce qui explique le soutien de la gauche modérée au concept de développement durable et encore moins d'un "totalitarisme vert " comme le craignent ceux qui désirent «restaurer la souveraineté de l'Amérique ${ }^{49}$ ", mais d'une conception de l'État «Pygmalion de la société: son sculpteur, son animateur et son promoteur tout à la fois ${ }^{50}$ » ou de l'État propulsif qui « se sert du droit pour agir sur des systèmes sociaux autonomes (économie, écologie, culture, éducation, etc.) en vue de les orienter dans un sens jugé plus conforme à l'intérêt général que le développement spontané des comportements guidés par le marchés1 ", qui peut aisément se concilier avec l'idéologie libérale ou avec une approche néo-colbertiste - d'où l'acceptation du développement durable par la plupart des gouvernements de droite ou du centre et son rejet par les mouvements d'extrême droite ou libertariens.

Cette compatibilité du développement durable avec le système capitaliste explique également le rejet de l'économie verte par certains États comme l'Équateur, retrouvant le souffle contestataire de la théorie de la dependencia ${ }^{52}$ pour dénoncer un complot impérialiste des États développés pour continuer à piller les ressources naturelles des pays du

48. Voir François-Xavier Merrien, Raphaël Parchet et Antoine Kernen, L'État social. Une perspective internationale, Paris, Armand Colin, 2005, p. 265-273; symptomatique de cette référence au Welfare State est le sous-titre du rapport du professeur Edward B. Barbier, Rethinking the Economic Recovery: A Global Green New Deal, PNUE, 2009, disponible sur http://www.sustainable-innovations.org/ GE/UNEP $|\% 20| \% 5$ B2009 $\mid \% 5 \mathrm{D} \backslash \% 20 \mathrm{~A} \backslash \%$ 2oglobal $\mid \%$ 2ogreen $\mid \% 20$ ew $\backslash \%$ 2odeal.pdf [consulté le 2 juin 2012].

49. Voir http://www.una-okc.org/anti-UN_voices.htm [consulté le 19 juin 2012]; l'auteur est redevable pour cette source à sa collègue Alynna Lyon, université du New Hampshire, qui en fit état lors de sa communication « Rethorics and Reality: the Domestic Politics of America », lors du congrès annuel du Conseil universitaire sur le système des Nations unies (ACUNS), New York, 14 juin 2012.

50. Georges Abi-Saab, "Cours général de droit international public», Recueil des cours de l'Académie de droit international, t. CCVII, 1987, p. 86.

51. Charles-Albert Morand, Le Droit néo-moderne des politiques publiques, Paris, LGDJ, 1999, p. 71.

52. La théorie de la dépendance est apparue à la fin des années 1960 sous la plume d'intellectuels latino-américains dénonçant le sous-développement des pays du Sud comme structurellement entretenu par ceux du Nord, Voir Cristóbal Kay, Latin American Theories of Development and Underdevelopment, Londres-New York, Routledge, 1989, surtout p. 125-162; pour une version actuelle, voir Oswaldo de Rivero, Le Mythe du développement. Les économies non viables du XxI siècle, 
Sud tout en refusant un réel libre-échange puisqu'ils restreignent l'accès des produits du Sud à leurs marchés grâce à un protectionnisme repeint en vert. Une semblable récusation de l'« économie verte» est manifestée par la mouvance altermondialiste ${ }^{53}$ pour laquelle les problèmes de la planète proviennent du capitalisme. Aussi surprenant à première vue est le désaveu du développement durable par une frange significative d'écologistes néo-malthusiens ayant une approche globalisante des questions environnementales et sociales, qui remettent en cause la logique productiviste et prêchent pour la décroissance, l'autolimitation de la consommation matérielle conformément à la formule Ghandi, selon qui «la terre produit assez pour satisfaire les besoins de chacun, mais non pour satisfaire la cupidité ${ }^{4}$ », voire le partage du travail, puisque «le temps de l'abondance est terminé, que le temps de l'État providence est terminé ${ }^{55}$ » Néanmoins, la remise en cause du mode de vie consumériste qui renoue avec la célébration épicurienne de la vie frugale et de la discipline va évidemment à rebours de l'évolution du monde des cinq derniers siècles et des aspirations d'une population mondiale aujourd'hui majoritairement urbaine.

Raymond Robitaille (trad.), Paris-Montréal, Éditions Charles Léopold Mayer Éditions Écosociété, 2003.

53. Voir notamment Bertrand Méheust, La Politique de l'oxymore. Comment ceux qui nous gouvernent nous masquent la réalité du monde, Paris, La Découverte, 2009; Romain Feli, Les Deux Âmes de l'écologie. Une critique du développement durable, Paris, L'Harmattan, 2008 ; Stéphane Bonnevault, Développement insoutenable: pour une conscience écologique et sociale, Bellecombe-en-Bauges, Éditions du Croquant, 2003. Voir aussi le site (en français): http://nogreeneconomy.org. Voir enfin Tadzio Mueller et Nicola Bullard, «Beyond the "Green Economy": System Change, Not Climate Change? Global Movements for Climate Justice in a Fracturing World», communication présentée à la conférence de l'Institut de recherches des Nations unies pour le développement social (UNRISD), Green Economy and Sustainable Development: Bringing Back the Social Dimension, Genève, 10-11 octobre 2011, disponible sur http://www.unrisd.org/80256B42004CCC77/ (httpinfoFiles)/67569F881A578E76C1257942005654B2/\$file/3-2 $\ \% 20 B u l l a r d \backslash$ \%20and $\mid \% 20$ Muller.pdf [consulté le 29 juin 2012].

54. Cité par Catherine de Silguy, Sagesse de la nature et folies des hommes, Paris, Le Cherche Midi, 2005, p. 128.

55. Antoine Waechter, «La naissance de l'écologie politique», Écho nature, $\mathrm{n}^{\circ}$ 3, aoûtseptembre 2005, p. 17. 


\section{L'éducation et la participation au cour du développement durable}

La déclaration réaffirme "que l'accès universel à un enseignement de qualité à tous les niveaux est une condition essentielle du développement durable, de l'élimination de la pauvreté, de l'égalité des sexes, de l'autonomisation des femmes et du développement humain, ainsi que de la réalisation des objectifs de développement arrêtés sur le plan international ${ }^{56}$ ». Ces objectifs de développement durable devront être atteints grâce à la " participation active et concrète des organes législatifs et judiciaires aux niveaux régional, national et sous-régional ainsi que de tous les grands groupes: femmes, enfants et jeunes, peuples autochtones, organisations non gouvernementales, autorités locales, travailleurs et syndicats, entreprises et secteurs d'activité, monde scientifique et technique et agriculteurs, ainsi que d'autres parties prenantes, notamment les collectivités locales, les groupes de bénévoles et les fondations, les migrants, les familles, les personnes âgées et les personnes handicapées ${ }^{57}$ ». Ainsi que l'écrivent Gilles Bœuf et Jean-Patrick Le Duc, dirigeants du Muséum national d'histoire naturelle, "à travers ces énumérations, le système atteint certaines limites, car les fonctionnaires hommes, ni jeunes, ni vieux, ni scientifiques et ni handicapés gardent eux aussi, un rôle important à jouer ${ }^{58} »$. En tout état de cause, le souhait de faire participer tous et chacun - ce qui implique une habilitation (empowerment) des plus vulnérables et des plus marginalisés ${ }^{59}$ - confirme que la profession de foi démocrate de la déclaration n'est pas qu'une figure de style. En dépit du caractère juridiquement non contraignant de L'avenir que nous voulons, les ressortissants des États à régime autoritaire ou totalitaire pourront se prévaloir de ce principe de participation plus détaillé que dans la déclaration de 1992.

56. L'avenir que nous voulons, op. cit., $\$ 229$; le $\$ 230$ poursuit: «Nous sommes déterminés à doter nos systèmes éducatifs des moyens de mieux préparer les jeunes à promouvoir le développement durable, notamment en améliorant la formation des enseignants, en mettant au point des programmes scolaires abordant les questions liées à la durabilité et des programmes de formation préparant les étudiants à des carrières dans des domaines en rapport avec la durabilité, et en faisant un usage plus efficace des technologies de l'information et de la communication afin d'améliorer l'apprentissage.»

57. Ibid., $\$ 43$.

58. http://vigienature.mnhn.fr/blog/ne-pas-manquer/ le-bilan-de-rio-2o-premiere- analyse-du-museum-national-dhistoire-natuelle. htm, 25 juin 2012 [consulté le 27 juin 2012].

59. Par exemple pour les femmes, voir L'avenir que nous voulons, op. cit., $\$ 238$. 
La déclaration pérennise et amplifie le concept de développement durable et ses apports ne sont pas insignifiants, en revanche, l'enrichissement annoncé du concept par l'incorporation de l'«économie verte » n'a pas été au rendez-vous, cette dernière notion manquant de finir en charpie lors des ultimes négociations.

\subsubsection{Avis de tempête sur l'économie verte}

Dix-huit paragraphes sont consacrés à l'« économie verte dans le contexte du développement durable et de l'élimination de la pauvreté", tout en s'efforçant soigneusement de ne pas la définir. Il est cependant indiqué que «les politiques de promotion d'une économie verte dans le contexte du développement durable et de l'élimination de la pauvreté » devraient notamment :

- être conformes au droit international ;

- respecter la souveraineté de chaque pays...;

- s'appuyer sur un environnement porteur et sur des institutions qui fonctionnent à tous les niveaux...;

- promouvoir une croissance économique durable et sans exclusive...;

- prendre en compte les besoins des pays en développement...;

- renforcer la coopération internationale...;

- ne pas constituer un moyen de discrimination arbitraire ou injustifiable ni une restriction déguisée aux échanges internationaux, éviter les actions unilatérales visant à résoudre les grands problèmes écologiques au-delà de la juridiction du pays importateur, et veiller à ce que les mesures de lutte contre les problèmes environnementaux transfrontières ou mondiaux soient, autant que possible, fondées sur consensus international ;

- contribuer à combler les lacunes entre pays développés et pays en développement...;

- améliorer le bien-être des peuples et des communautés autochtones, d'autres populations locales et traditionnelles et des minorités ethniques...;

- améliorer le bien-être des femmes, des enfants, des jeunes, des personnes handicapées, des petits exploitants et agriculteurs de subsistance, des pêcheurs et des ouvriers des petites et moyennes entreprises, et améliorer les moyens de subsistance et l'autonomisation des groupes indigents et vulnérables...;

- tirer parti de tout le potentiel qu'offrent les femmes et les hommes et assurer leur contribution à égalité;

- promouvoir, dans les pays en développement, des activités productives qui contribuent à éliminer la pauvreté ;

- répondre aux préoccupations en matière d'inégalités et promouvoir l'intégration sociale, et notamment une protection sociale minimale;

- promouvoir des modes de consommation et de production durables; 
- poursuivre les efforts déployés pour mettre sur pied des approches pour un développement équitable et sans exclusive ${ }^{60} \ldots$

Cette litanie d'intentions convenues ne renseigne guère sur ce que pourrait être une "économie verte", il faut donc chercher dans les travaux antérieurs ce que devrait recouvrir ce syntagme, ainsi que dans les « domaines thématiques et questions transversales » de la déclaration d'éventuelles illustrations.

\subsubsection{Un concept vidé de sa substance}

L'introduction de l'économie verte devait être l'un des principaux apports du sommet Rio + 20, mais la montagne accoucha finalement d'une souris, car les États n'ont réussi à s'accorder ni sur ce que devait recouvrir l'économie verte, ni sur les investissements nécessaires à la transition de l'actuelle «économie brune » à l'économie verte.

\section{Désaccords sur le sens de l'économie verte}

Ayant consacré depuis plus de quatre ans maintes études sur le sujet, le programme des Nations unies pour l'environnement (PNUE) définit l'économie verte comme "une économie qui entraîne une amélioration du bien-être humain et de l'équité sociale tout en réduisant de manière significative les risques environnementaux et la pénurie des ressources ${ }^{61}$ ». Cette formulation simple et apparemment consensuelle n'a donc pas été retenue par la déclaration qui a préféré dresser un inventaire des contraintes qui devront peser sur les politiques de promotion d'une économie verte.

L'absence de définition est évidemment due à une absence d'accord, certains États appréhendant l'économie verte comme un moyen de maintenir un système économique qu'ils combattent, d'autres pays - et pas uniquement ceux du Sud - craignant qu'une réduction significative des risques n'entrave leur développement ou ne rende plus onéreuse leur politique environnementale. Déjà lors de la conférence de Durban (Afrique du Sud, 28 novembre-11 décembre 2011), «l'Inde, délaissant sa position conciliante naguère retenue et réclamant l'application du

60. L'avenir que nous voulons, op. cit., $\$ 58$, points $a$ à $p$; le $\$ 226$ de la déclaration signale l'adoption d'un cadre décennal de programmation concernant les modes de consommation et de production durables (Doc. ONU A/CONF.216/5), tout en précisant que les programmes qu'il contient ont un caractère facultatif.

61. Vers une économie verte: pour un développement durable et une éradication de la pauvreté. Synthèse à l'utilisation des décideurs, 2011, p. 2, disponible sur http: //www.unep.org/greeneconomy.htm [consulté le $1^{\text {er }}$ juin 2012]. 
principe d'équité, a annoncé sa volonté de rejeter tout nouvel accord juridiquement contraignant pour les États émergents, confirmant en cela son rôle de porte-voix de ce groupe hétéroclite de pays ${ }^{62}$ ». À Rio de Janeiro, l'Afrique du Sud, le Brésil, la Chine et la Russie ont rejoint l'Inde pour refuser de se voir imposer des contraintes susceptibles de nuire à leur croissance économique, tandis que le Canada et les ÉtatsUnis, de concert avec le G 77, se sont opposés à la réaffirmation de la responsabilité incombant aux entreprises de respecter les Droits de l'homme ${ }^{63}$.

\section{Désaccords sur la portée des efforts financiers}

Les signataires de la déclaration sont tous conscients que la transition vers une économie verte, même réduite au plus petit dénominateur commun, nécessite des investissements. À cette fin, chaque pays et les organismes de l'ONU, les institutions financières internationales et les autres organisations internationales sont invités à participer à la mise en place de politiques favorables à l'économie verte. De même, le secteur privé (milieux d'affaires ou d'industrie, mais aussi coopératives et microentreprises ${ }^{64}$ ) est appelé à y prendre part. La déclaration encourage «les partenariats - notamment entre les secteurs public et privé , existants et nouveaux, à mobiliser un financement public complété par des fonds du secteur privé, en tenant compte, le cas échéant, des intérêts des populations locales et des communautés autochtones ${ }^{65}$ ». Les sommes à collecter sont importantes puisque le PNUE prône «la nécessité économique et sociale d'investir $2 \%$ du PIB mondial dans le verdissement de dix secteurs cruciaux de l'économie [agriculture, logement, énergie (approvisionnement), pêche, foresterie, industrie, transport, tourisme, déchets, eau] afin de réorienter le développement et de canaliser les flux de capitaux publics et privés vers l'utilisation

62. Roland Séroussi, op. cit., p. 149.

63. Voir Human Rights Watch, http://www.hrw.org/fr/news/2012/06/22/ rio-le-document-final-amoindri-par-les-opposants-aux-droits-humains.htm [consulté le 30 juin 2012].

64. L'avenir que nous voulons, op. cit., $\$ 62,66,68-70$.

65. Ibid., $\$ 71$. 
rationnelle des ressources et la réduction des émissions de carbone ${ }^{66}$ ", mais cet objectif ne figure pas dans la déclaration.

Pour sa part, l'Organisation de coopération et de développement économiques (OCDE) considère que les outils pour la mise en place d'une « croissance verte» diffèrent selon le type d'économie :

- les pays développés doivent privilégier la recherche-développement dans l'innovation technologique, les investissements dans les infrastructures à faible intensité de carbone et la tarification de l'externalité au moyen d'instruments fondés sur le marché;

- les pays en développement doivent surtout réduire les infrastructures à forte intensité de carbone, promouvoir des technologies efficientes en termes de consommation d'énergie et de matériaux, renforcer les pouvoirs publics, et développer, diffuser et transférer des technologies;

- et les pays les moins avancés doivent éviter le régime d'accès libre aux ressources naturelles, accroître la productivité de l'utilisation nette des ressources, évaluer les risques pour le climat des politiques, plans et programmes nationaux, et investir en infrastructures afin de faciliter l'accès au marché ${ }^{67}$.

Ces préconisations ne se retrouvent pas non plus dans la déclaration rédigée en termes généraux et parfois vagues, presque sans prise en considération du niveau de développement économique ${ }^{68}$.

66. Achim Steiner, préface à Vers une économie verte..., op. cit., p. v; les seuls objectifs chiffrés en matière de financement se trouvent au $\$ 258$, où les signataires invitent notamment «les pays développés qui ne l'ont pas encore fait [c'est-à-dire la grande majorité d'entre eux] à s'attacher plus concrètement à atteindre l'objectif fixé, à savoir consacrer $0,7 \%$ de leur PNB à l'[aide publique au développement] en faveur des pays en développement, ainsi que l'objectif plus spécifique de $0,15 \%$ à $0,20 \% \mathrm{du}$ PNB pour les pays moins avancés, comme ils se sont engagés à le faire ».

67. Voir Nathalie Girouard, "Un cadre d'action pour une croissance verte », dans Conseil économique pour le développement durable, Paroles d'économistes, 2011, p. 13-15, http://www.developpement-durable.gouv.fr/IMG/pdf/o2-6.pdf [consulté le $1^{\text {er }}$ juin 2012].

68. Toutefois le $\$ 269$ souligne «l'importance du transfert de technologie pour les pays en développement [...] à des conditions favorables, y compris à des conditions de faveur ou préférentielles convenues d'un commun accord», et les spécificités de certains territoires sont pris en compte: petits États insulaires en développement $(\$ 178-180)$, pays les moins avancés ( $\$ 181)$, pays en développement sans littoral $(\$ 182)$ et Afrique ( $\$ 183-184)$. Des actions régionales sont recommandées, «en particulier dans la région arabe, en Amérique latine et dans les Caraïbes, ainsi que dans la région de l'Asie et du Pacifique» ( $\$ 185)$, mais il ne s'agit que de réitérer des engagements préalablement pris. 
D'un autre côté, il convient de noter que les pays de l'OCDE se sont opposés à la création d'un fonds de 30 milliards de dollars pour soutenir le développement durable demandée par la Chine et le $\mathrm{G} 77^{69}$.

\subsubsection{Un faible impact de l'économie verte sur les questions transversales}

Les travaux du PNUE ou de l'OCDE laissent peu de traces dans le traitement des différents secteurs économiques où des solutions éprouvées relevant de la coopération internationale classique ou de bonnes pratiques aux niveaux national et infra-étatique ont été préférées.

En matière de sécurité alimentaire et de nutrition, la déclaration se contente de réitérer les engagements déjà pris au sujet du «droit de chaque être humain d'avoir accès à des aliments sains et nutritifs en quantité suffisante conformément au droit à une alimentation adéquate et au droit fondamental de chacun d'être à l'abri de la faim ». Les dix paragraphes qui suivent ce rappel se satisfont de recommandations relatives au secteur agricole, à la pêche ou à l'aquaculture, à la recherche agricole, aux comités de la sécurité alimentaire mondiale et pour la gouvernance responsable du régime de propriété foncière, des pêches et des forêts et en matière de prix alimentaires ${ }^{70}$, pour finir par l'éloge du libre-échange ${ }^{71}$. La propriété intellectuelle est tempérée dans les paragraphes relatifs à la santé, puisque les États s'engagent «à redoubler d'efforts pour permettre un accès universel aux services de prévention, de traitement, de soins et de soutien en matière de V.I.H., et pour lutter contre la transmission du virus de la mère à l'enfant, ainsi que pour relancer ou renforcer le combat contre le paludisme, la tuberculose et les maladies tropicales » et réaffirment « le droit de se prévaloir pleinement des dispositions de l'accord sur les aspects des droits de propriété intellectuelle qui touchent au commerce (ADPIC), de la déclaration de Doha relative à l'accord sur les ADPIC [...] qui prévoient des

69. Lucien Chabasson, «Rio +20 ou le sommet de l'inaction », Diplomatie, $\mathrm{n}^{\circ} 58,2012$, p. 33.

70. L'avenir que nous voulons, op. cit., $\$ 108-117$.

71. Ibid., $\$ 118$; la déclaration se situe clairement dans une optique libérale, puisque le $\$ 281$ consacré au commerce proclame: «L'existence d'un système commercial multilatéral universel; réglementé, ouvert, non discriminatoire et équitable, assorti d'une véritable libéralisation des échanges, peut stimuler de façon déterminante la croissance économique et le développement dans le monde entier, au profit de tous les pays, quel que soit leur stade de développement, alors qu'ils progressent vers le développement durable.» 
assouplissements destinés à protéger la santé publique et, en particulier, à promouvoir l'accès aux médicaments pour tous ${ }^{72}$ ».

En matière d'énergie, alors même qu'on a pu faire allusion à l'exigence d'une véritable révolution du droit international de l'environnement ${ }^{73}$, aucun engagement concret n'est pris et si les signataires de la déclaration sont « conscients qu'il importe de faire une utilisation plus rationnelle de l'énergie et d'accroître la part des énergies renouvelables, des technologies moins polluantes et des techniques à haut rendement énergétique, pour parvenir au développement durable, y compris pour lutter contre les changements climatiques ${ }^{74}$ ", ils n'en tirent aucune conclusion chiffrée. De même, les deux paragraphes consacrés aux modes de transport viables se bornent à une énumération de poncifs qui n'engagent à rien ${ }^{75}$.

Une des rares innovations de L'avenir que nous voulons par rapport aux textes adoptés depuis vingt ans est la mention de la nécessité de soutenir « les activités liées développement durable du tourisme » et de renforcer les capacités « qui favorisent la connaissance de l'environnement, conservent et préservent l'environnement, respectent la vie sauvage, la flore, la diversité biologique, les écosystèmes et la diversité culturelle, et améliorent les conditions de vie et les sources de revenu des populations locales en protégeant leur économie, ainsi que l'environnement et le milieu naturel dans son ensemble ${ }^{76} »$. Pourtant peu médiatisés dans les conférences environnementales, l'écotourisme et le tourisme culturel, qui n'étaient pour autant pas dépourvus de textes internationaux, fussent-ils de droit programmatoire ${ }^{77}$, sont ainsi consacrés comme des véhicules du développement durable ${ }^{78}$.

La préoccupation pour les questions urbanistiques est, elle, plus classiquement présente dans les conférences internationales environnementales, notamment par le biais du programme Action 21. La déclaration

72. Ibid., $₫ 140$ et 142 .

73. Voir Caroline Migazzi, «La sécurité énergétique et le droit international de l'environnement ", dans Stéphane Doumbbé-Billé (dir.), Défis énergétiques et droit international, Bruxelles, Larcier, 2011, p. 303-308.

74. L'avenir que nous voulons, op. cit., $\$ 128$.

75. Ibid., $\$ 132-133$.

76. Ibid., $\$ 130$.

77. Sous les auspices de l'Organisation mondiale du tourisme (OMT), du PNUE et de l'UNESCO, fut adoptée en 1995 une Charte du tourisme durable, consultable sur http://www.insula.org/tourism/version.htm. L'OMT a adopté en 1999 un Code mondial d'éthique du tourisme, disponible sur http://www.world-tourism.org/ code_ethics/eng.html.

78. Voir Rémy Knafou, "Tourisme et "développement durable" ", dans Yvette Veyret (dir.), Le Développement durable, Paris, Sedes, 2007, p. 178-192. 
préconise « une stratégie globale en matière d'urbanisme et d'établissements humains qui prévoit des logements et des infrastructures d'un coût abordable et qui privilégie l'assainissement des quartiers insalubres et la rénovation urbaine » et propose de "protéger le patrimoine naturel et culturel que constituent les établissements humains, [de] restaurer les quartiers historiques et [de] rénover le centre des villes ${ }^{79}$ ".

Quant à la réduction des risques, la déclaration appelle « à prendre de toute urgence des mesures visant à atténuer les risques de catastrophe et à accroître la résilience ", ce qui implique la mise en place de systèmes d'alerte rapide, le partage entre États des connaissances et de l'information, notamment des informations géospatiales, l'assistance technique et le transfert de technologie. La déclaration prévoit de "garantir, d’ici à 2020, une gestion rationnelle des produits chimiques, tout au long de leur cycle de vie, et des déchets dangereux, de façon à réduire au minimum les effets néfastes graves sur la santé humaine et l'environnement, conformément au Plan de mise en œuvre de Johannesburg» et incite les États et régions à appliquer une "gestion rationnelle des produits chimiques et des déchets à tous les niveaux qui réponde de manière effective, efficiente, cohérente et coordonnée aux questions et défis existants et qui se font jour». Les États s'engagent à "réduire, réutiliser et recycler [les trois $\mathrm{R}$ ] davantage les déchets et à en améliorer la valorisation énergétique » et promeuvent «la mise au point de solutions écologiques et plus sûres propres à remplacer les substances chimiques dans les produits et les procédés ». Enfin, après avoir souligné l'importance économique des activités extractives et reconnu le droit souverain des pays "d'exploiter leurs ressources minérales en fonction de leurs priorités nationales", la déclaration plaide pour la mise en place de "garanties concrètes visant à réduire les incidences négatives sur la société et l'environnement et à préserver la biodiversité et les écosystèmes, notamment après la fermeture des mines » et appelle « les gouvernements et les entreprises à s'efforcer de continuer d'accroître la responsabilité et la transparence ainsi que l'efficacité des mécanismes existants destinés à prévenir les flux financiers illicites provenant des activités extractives ${ }^{80}$ ".

La déclaration souhaite la "promotion du plein emploi et de l'emploi productif, du travail décent pour tous et de la protection sociale", mais elle insiste surtout sur ce qui devrait être fait plutôt que de s'engager réellement sur des propositions concrètes, sauf dans le dernier

79. L'avenir que nous voulons, op. cit., $\$ 134$.

80. Ibid., \$186-187, 213, 218, 220, 227-228. 
paragraphe qui, paradoxalement, met le plus en jeu le contrôle du territoire et donc la souveraineté, puisqu'il est demandé «aux États de promouvoir et de défendre efficacement les droits de l'homme et les libertés fondamentales de tous les migrants, en particulier les femmes et les enfants, quel qu'en soit le statut migratoire, et de traiter la questions des migrations internationales par la voie de la coopération et du dialogue au plan international, régional ou bilatéral et d'une manière globale et équilibrée, en tenant compte du rôle et des responsabilités des pays d'origine, de transit et de destination dans la promotion et la défense des droits de l'homme de tous les migrants et en évitant les démarches qui pourraient rendre ces derniers encore plus vulnérables ${ }^{81} »$.

Malgré le travail accompli par le PNUE et l'OCDE, l'économie verte qui devait être le moteur d'une version rénovée du développement durable est donc loin d'être consacrée. Certes, le terme figure dans la déclaration mais son contenu a été passablement affadi. Il en va de même de la gouvernance du développement durable puisque les espoirs de son renforcement ont également été déçus.

\section{La réaction souverainiste du sommet Rio +20}

Nombreux étaient ceux qui escomptaient que la conférence de 2012 déboucherait sur de notables progrès en matière de gouvernance, voire sur l'adoption de traités - ou du moins le lancement de conférences diplomatiques - concernant de multiples sujets. C'était s'illusionner sur la prétendue entrée du monde dans une ère "post-westphalienne » de souverainetés partagées. Les États acceptent le dialogue avec les organisations intergouvernementales, les sociétés transnationales et les ONG, mais refusent d'abandonner leur compétence décisionnelle. En conséquence, la gouvernance mondiale du développement durable est enterrée et certains dossiers brûlants ont été ajournés.

\subsection{Requiem carioca pour la gouvernance mondiale du développement durable}

L'affaire semblait plutôt bien engagée puisque, un an avant l'ouverture de la conférence, le secrétariat de l'ONU lançait une consultation télématique de la société civile, et de multiples réunions d'ONG, de think tanks et d'experts firent remonter leurs cahiers de doléances. Sur place, l'initiative brésilienne Dialogues de Rio a recueilli elle aussi, 
grâce à une plateforme en ligne, les avis de la société civile sur les thèmes des villes durables, de l'économie du développement durable, du développement durable comme réponse aux crises économiques et financières, de l'énergie, du chômage, de l'eau, de la sécurité alimentaire, de la lutte contre la pauvreté, des forêts et des océans. Le million de contributions reçues ont servi de base à quatre jours de débats auxquels des milliers de personnes ont participé ${ }^{82}$. Pourtant les États n'ont que très peu retenu ces propositions « d'en bas » et la gouvernance mondiale du développement durable telle qu'elle était envisagée par beaucoup s'efface devant un renouveau de l'intergouvernemental, le dispositif institutionnel étant des plus classiques.

\subsubsection{Les deux faces de la gouvernance mondiale}

L'expression "gouvernance mondiale » décrit à la fois ce qui est déjà et ce qui devrait être. En tant que critère explicatif, la gouvernance mondiale dépeint les relations internationales comme caractérisée par « une complexité croissante en raison du nombre de plus en plus élevé d'acteurs impliqués, une érosion partielle des souverainetés étatiques, une pluralité des dispositifs de pilotage plus ou moins bien coordonnés, une série d'arrangements formels et/ou informels toujours en négociation »: «Le monde de la gouvernance est celui d'une intégration balbutiante, de régulations dispersées et concurrentes, mais aussi celui d'une idéologie de l'efficacité qui dicte ses politiques (la "bonne gouvernance") et fonctionne, le cas échéant, comme une procédure d'exclusion ${ }^{83}$.» Force est de constater que, structure d'accueil, l'ONU permet effectivement une certaine participation de l'opinion publique mondiale. Depuis la conférence de Rio de 1992, les ONG sont associées aux négociations par la tenue de forums parallèles et cherchent à influencer les délégations diplomatiques en appelant leur attention sur des intérêts catégoriels. Non seulement les ONG peuvent se faire entendre et, parfois, influer sur les décisions prises, mais elles peuvent aussi participer

82. Voir Benoît Théau, De la déception aux nouveaux espoirs, p. 3, http:// www.positive-rio.tv/uploads/ckfinder/files/Bilan|\%2oRio+20_V2.pdf [consulté le 26 août 2012].

83. Guillaume Devin et Marie-Claude Smouts, Les Organisations internationales, Paris, Armand Colin, 2011, p. 118-119 [notes omises]. 
à la mise en œuvre d'un traité ${ }^{84}$. Cependant, force est de constater que ces ONG sont très majoritairement le reflet de subdivisions des sociétés occidentales et que les priorités qu'elles parviennent à faire adopter sont souvent celles des lobbies occidentaux les plus médiatiquement développés. D’autres groupes d'intérêt qualifiés de "parties prenantes", les sociétés transnationales ès-qualité ou, à travers des regroupements comme le World Business Council for Sustainable Development, voire des ONG ou think tanks stipendiés, sont également présents, de façon formelle ou officieuse, dans les conférences diplomatiques ou les conseils scientifiques et comités de suivi de conventions spécifiques. Cette gouvernance réellement existante n'est pas remise en cause. Au contraire, la déclaration affiche la détermination de la communauté internationale à renforcer le dispositif institutionnel du développement durable pour :

- encourager «l'intégration équilibrée des trois dimensions»;

- mettre « en exergue l'importance des liens existant entre les principaux problèmes et défis et la nécessité de les aborder systématiquement à tous les niveaux pertinents »;

- renforcer la cohérence et accroître "l'efficacité, l'efficience et la transparence»;

- inciter à une "participation pleine et effective de tous les pays aux processus de décision »;

- "mobiliser les dirigeants politiques de haut niveau ";

- stimuler les échanges entre scientifiques et décideurs, augmenter la participation de la société civile et favoriser «l'examen et le bilan des progrès réalisés dans la concrétisation de tous les engagements pris en matière de développement durable».

Le tout est censé reposer "sur une approche pragmatique et axée sur les résultats prenant dûment en considération toutes les questions intersectorielles pertinentes en vue de contribuer à la réalisation du développement durable ${ }^{85}$ ».

84. Par exemple, l'UICN contribue à la définition des espèces protégées par la convention de Washington du 3 mars 1973 sur le commerce international des espèces de faune et de flore sauvages menacées d'extinction, communément appelée CITES (Convention on International Trade of Endangered Species), dont le texte est partiellement reproduit dans Laurence Boisson de Chazournes, Richard Desgagné, Makane M. Mbengue et Cesare Romano, op. cit., p. 105-113.

85. L'avenir que nous voulons, op. cit., $\$ 76$, points $a$ à $i$. 
En ce qui concerne la gouvernance telle qu'elle devrait être dans l'esprit de ses thuriféraires ${ }^{86}$, les États, les organisations internationales et le secteur privé marchand et non marchand seraient placés pratiquement sur un pied d'égalité sous la direction de l'ONU, compte tenu de son magistère moral, puisque l'Organisation mondiale, représentation institutionnelle de la société internationale, est le seul forum légitime où peuvent être discutées les grandes orientations de "l'ère de la mondialité ${ }^{87}$ ".

Or, en vertu du principe de subsidiarité, l'ONU prend déjà en charge, à divers degrés, nombre de questions que les États ou les autres organisations intergouvernementales ne peuvent traiter, car elle est le seul acteur à avoir une vision globale des problèmes. Cependant, comme le système onusien, malgré ce que laisse penser son organigramme, est, de fait, peu centralisé $^{88}$, il n'arrive pas toujours à mettre en œuvre des politiques cohérentes, sans parler des cas où les politiques de ses institutions sont contradictoires comme, par exemple, les programmes d'ajustement structurels du Fonds monétaire international (FMI) et les plans d'action du PNUD. En outre, la prolifération, à l'extérieur du système onusien, d'institutions due à la multiplication de secrétariats créés par différents traités environnementaux ${ }^{89}$ et donc l'absence d'une institution centrale efficace et suffisamment financée n'aident guère. D'autant que les dizaines de secrétariats mis en place par les différentes conventions n'ont pas de relations systématiques entre eux et peuvent même pousser dans des directions différentes. Pour compliquer le tout, la Banque mondiale ou le FMI mettent en œuvre leur propre conception du développement

86. «Le conseiller du secrétaire général de la conférence de Stockholm de 1972 (Maurice Strong) était un certain $\mathrm{D}^{\mathrm{r}} \mathrm{Jim}$ MacNeill, chercheur et consultant canadien, qui devint lui-même secrétaire général de la commission Brundtland, et le principal auteur du fameux rapport de 1997. En 1991 [...], il écrit avec deux co-auteurs un ouvrage intitulé Beyond Interdependence, qui précise le lien entre développement durable et gouvernance mondiale [...] il parle, pour 2012, d'un nouveau partenariat mondial sous forme d'un système politique international revitalisé par un Conseil de la terre, sorte de conseil de sécurité à mandat élargi »(Claude Revel, op. cit., p. 23).

87. «L'ère de la mondialité signifie que la société humaine a pris conscience de son existence en tant que communauté mondiale, et non plus en tant qu'ensembles multiples, juxtaposés ou interdépendants, de groupements humains, peuples, nations, uniques et indépendants, dont l'histoire récente aurait intensifié et approfondi les relations de domination-dépendance et de coopération " (Riccardo Petrella, Le Bien commun. Éloge de la solidarité, Lausanne, Page Deux, 1997, p. 99-100).

88. D'où des chevauchements de compétences, voire des programmes-doublons en dépit de moyens financiers réduits. Voir Maurice Bertrand, L'ONU, Paris, La Découverte, 1995, p. 60-67.

89. Voir Jean-Marc Lavieille (dir.), Conventions de protection de l'environnement. Secrétariats, conférences des parties, comités d'experts, Limoges, PULIM, 1999. 
durable via leur politique de conditionnalité écologique ${ }^{90}$. La direction des affaires du monde par l'ONU relève donc du fantasme davantage que de l'observation des faits.

Un fort courant d'opinion souhaitait que le sommet Rio + 20 transformât la gouvernance existante en une gouvernance rêvée. Il n'a pas été entendu.

\subsubsection{Le retour de l'intergouvernemental}

Le projet de création d'une organisation mondiale de l'environnement faisant pendant à l'Organisation mondiale du commerce (OMC) se heurtant à l'opposition tant des États-Unis et de certains États occidentaux que des pays émergents ${ }^{91}$, cette idée, dont la France s'était fait le héraut sous la présidence de Jacques Chirac et au début du quinquennat de Nicolas Sarkozy, a été abandonné au profit d'un affermissement du PNUE, lequel aurait dû absorber la CDD, mais finalement la déclaration se limite à "renforcer" le PNUE, qui ne compte actuellement que 58 États membres, et qui bénéficiera de l'adhésion universelle des membres de l'ONU à son Conseil d'administration.

Les ressources financières du PNUE seront augmentées via une allocation accrue du budget ordinaire de l'ONU. La capacité du PNUE à «s'acquitter de son mandat coordination au sein du système des Nations unies" sera raffermie, tandis que seront rationalisées "progressivement les fonctions du siège à Nairobi » et que sera renforcée "sa présence régionale de manière à aider les pays, à leur demande, à mettre en ouvre leurs politiques environnementales nationales, en collaborant étroitement avec d'autres entités compétentes du système des Nations unies ${ }^{92}$ ".

Contrairement à ce qu'avait un temps envisagé la France, il n'y a pas de modification de l'intitulé du Conseil économique et social (Écosoc) pour $\mathrm{y}$ adjoindre le qualificatif «environnemental » - à l'instar de la révision constitutionnelle de 2008 - car cela eût impliqué une modification de la Charte de l'ONU, néanmoins l'Écosoc devrait être renforcé « en tant qu'organe principal chargé du suivi intégré et coordonné des conclusions issues de l'ensemble des principaux sommets et conférences

90. Voir Philippe Sands, «Environmental Protection in the Twenty-First Century: Sustainable Development and International Law», dans Richard L. Revesz, Philippe Sands et Richard B. Stewart (dir.), Environmental Law, the Economy and Sustainable Development, Cambridge University Press, 2000, p. 394.

91. Voir Lucien Chabasson, op. cit., p. 34.

92. L'avenir que nous voulons, op. cit., $\$ 88$, points $a$ à $h$. 
des Nations unies consacrés aux questions économiques, sociales et environnementales et aux questions connexes ${ }^{93}$ ». En revanche, il est décidé le remplacement à terme de la CDD par une « instance politique de haut niveau», laquelle pourrait :

- exercer une action mobilisatrice...;

- améliorer l'intégration des trois dimensions du développement durable de manière holistique et intersectorielle à tous les niveaux;

- constituer une tribune dynamique...;

- suivre et passer en revue les progrès accomplis dans la concrétisation des engagements pris [de Rio 1992 à Rio + 20];

- encourager la participation [...] des organismes, fonds et programmes des Nations unies et convier à participer [...] d'autres institutions financières et commerciales multilatérales pertinentes et organes conventionnels...;

- améliorer la coopération et la coordination au sein du système des Nations unies...;

- promouvoir la transparence...;

- promouvoir le partage des meilleures pratiques...;

- promouvoir la cohérence et la coordination des politiques de développement durable à l'échelle du système ;

- intensifier les échanges entre scientifiques et décideurs;

- [et] étayer la prise de décisions fondées sur les faits à tous les niveaux et contribuer à l'intensification des efforts engagés pour renforcer les capacités en matière de collecte et d'analyse des données dans les pays en développement $^{94}$.

Le sommet Rio + 20, loin d'opérer une refondation de la gouvernance, améliore simplement l'existant et dans un sens purement intergouvernemental, signifiant ainsi que nous vivons dans un « monde d'États-nations où toute dérogation de souveraineté aux organisations du système des Nations Unies paraît dangereuse et inacceptable ${ }^{95}$ ".

La déclaration donne d'ailleurs en exemple la pratique intergouvernementale. D'une part, elle reconnaît «qu'il importe d'associer plus étroitement et plus largement les pays en développement à la prise de décisions et au processus de normalisation internationaux dans le domaine économique » et prend acte « des décisions importantes qui ont récemment été prises en ce qui concerne la réforme des mécanisme de gouvernance, des quotes-parts et des droits de vote au sein des institutions de Bretton Woods, de manière à mieux refléter les réalités actuelles,

\section{Ibid., $\$ 83$.}

94. Ibid., $\$ 85$, points $a$ à $l$.

95. Lettre de l'IDDRI, $\mathrm{n}^{\circ}$ 31, juillet 2012, http://www.iddri.org/Iddri/Lettre-de-1-Iddri/ Rio+20-et-apres.htm [consulté le 9 août 2012]. 
à donner davantage voix au chapitre aux pays en développement et à leur assurer une participation accrue $e^{96} »$.

D'autre part, à l'instar des activités déjà engagées pour accroître les synergies entre la convention de Bâle du 22 mars 1989 sur le contrôle des mouvements transfrontières des déchets dangereux et de leur élimination $^{97}$, la convention de Rotterdam du 11 septembre 1998 sur la procédure de consentement préalable en connaissance de cause applicable à certains produits chimiques et pesticides dangereux qui font l'objet d'un commerce international ${ }^{98}$ et la convention de Stockholm du 22 mai 2001 sur les polluants organiques persistants ${ }^{99}$, la déclaration encourage «les parties aux accords multilatéraux sur l'environnement à envisager de nouvelles mesures dans le cadre de ces conventions et d'autres instruments, selon qu'il convient, pour promouvoir la cohérence des politiques à tous les niveaux requis, agir plus efficacement, réduire les chevauchements et doublons inutiles et renforcer la coordination et la coopération entre les accords multilatéraux sur l'environnement, y compris les trois Conventions de Rio, ainsi qu'avec le système des Nations unies sur le terrain». Enfin, bien que la Déclaration prenne soin de le dissocier du dispositif institutionnel de la gouvernance, il est prévu « un mécanisme intergouvernemental transparent et participatif [...], ouvert à toutes les parties prenantes afin de formuler des objectifs de développement durable de portée mondiale, devant être adoptés par l'Assemblée générale des Nations unies ${ }^{100} "$.

Amélioration des canaux de communication avec les acteurs non étatiques qui demeurent néanmoins tenus en lisière, renforcement de la cohérence des actions interétatiques au sein de la famille de l'ONU ou des accords multilatéraux sur l'environnement et montée en puissance du PNUE, le sommet de Rio + 20 n’a donc pas cédé aux sirènes « postwestphaliennes " mais était-il raisonnable d'espérer autre chose d'un club d'États? En revanche, il semblait réaliste de s'attendre à ce que les problèmes les plus importants portés à l'attention de la conférence reçoivent l'attention qu'ils méritent, or tel n'a pas toujours été le cas.

96. L'avenir que nous voulons, op. cit., $\$ 92$.

97. Texte partiellement reproduit dans Laurence Boisson de Chazournes, Richard Desgagné, Makane M. Mbengue et Cesare Romano, op. cit., p. 493-503.

98. Texte reproduit, sans ses annexes, ibid., p. 503-520.

99. Texte partiellement reproduit ibid., p. 521-534.

100. L'avenir que nous voulons, op. cit., $\$ 89$ et 248 . 


\subsection{Les omissions de la déclaration}

La déclaration fait l'impasse sur un certain nombre de sujets préoccupants parmi lesquels la protection du milieu marin, l'accès à l'eau et les déplacés environnementaux.

\subsubsection{La protection du milieu marin en haute mer et contre les pollutions provenant de la terre}

Alors que les dispositions sur la pêche sont abondantes ${ }^{101}$, la déclaration ne prend pas suffisamment en considération la protection du milieu marin et notamment de sa biodiversité.

La déclaration souhaite que la convention des Nations unies sur le droit de la mer «soit adoptée quasiment universellement par les États » et, de façon plus surprenante, prie «tous ses États parties de respecter pleinement les obligations que leur impose la convention ${ }^{102} »$ ! Dans la même veine, les États parties à l'accord de New York du 4 août 1995 aux fins d'application des dispositions de la convention des Nations unies sur le droit de la mer du 10 décembre 1982 relatives à la conservation et à la gestion des stocks de poissons dont les déplacements s'effectuent tant à l'intérieur qu'au-delà des zones économiques exclusives (stocks chevauchants) et des stocks de poissons grands migrateurs ${ }^{103}$ sont priés "d'appliquer pleinement cet instrument et de tenir pleinement compte des besoins particuliers des États en développement, conformément à la partie VII de l'accord ${ }^{104}$ ». Cependant, ce qui est notable n'est pas tant ce que contient L'avenir que nous voulons que ce qui a disparu lors des commissions préparatoires. À la demande des États-Unis, du Canada, du Japon, de la Russie et du Venezuela, le paragraphe visant «la protection et l'usage durable de la biodiversité marine au-delà des zones se trouvant sous juridiction nationale » a été retiré de la version finale de la déclaration ${ }^{105}$.

Les optimistes se satisferont toutefois que ce ne soit que partie remise, puisque que les signataires, «conscients de l'importance que revêtent la conservation et l'exploitation durable de la biodiversité marine dans les zones situées en dehors des juridictions nationales», prennent «note

101. Ibid., $\$ 168-158$.

102. Ibid., $\$ 159$.

103. Texte partiellement reproduit dans Laurence Boisson de Chazournes, Richard Desgagné, Makane M. Mbengue et Cesare Romano, op. cit., p. 249-257.

104. L'avenir que nous voulons, op. cit., $\$ 169$.

105. Le Monde, 21 juin 2012. 
des travaux menés par un groupe de travail spécial officieux [...] sous l'égide de l'Assemblée générale » et s'engagent à traiter de la question " notamment en prenant une décision sur l'élaboration d'un instrument international dans le cadre de la convention des Nations unies sur le droit de la mer ${ }^{106}$ » en 2014, année durant laquelle le mécanisme de notification et d'évaluation systématiques à l'échelle mondiale de l'état du milieu marin, $\mathrm{y}$ compris les aspects socioéconomiques ${ }^{107}$ doit rendre sa première évaluation intégrée à l'Assemblée générale ${ }^{108}$.

Alors que l'adoption d'un traité sur la pollution marine d'origine tellurique était espérée ${ }^{109}$, la déclaration se contente d'un engagement "à prendre des mesures en vue de réduire les effets de cette pollution " et «à agir pour réduire de façon importante les déchets marins d'ici à $2025^{110} \%$.

\subsubsection{L'accès à l'eau}

La déclaration se borne en la matière à des imprécations ${ }^{111}$, mais n'apporte rien de nouveau, alors que le droit international est particulièrement pauvre pour faire face aux problèmes d'accès à la ressource en eau et de la qualité de celle-ci. Si les États ont pu s'accorder sur le régime de navigation fluviale ${ }^{112}$, il n'y a pas de réglementation universelle. La convention d'Helsinki du 17 mars 1992 sur la protection et l'utilisation des cours d'eau trans-frontières et des lacs internationaux, complétée

106. L'avenir que nous voulons, op. cit., $\$ 162$.

107. Groupe d'experts créé par la résolution 58/240 de l'Assemblée générale, conformément au $\$ 36 b$ du plan de mise en œuvre de Johannesburg, voir http://www.un. org/Depts/los/global_reporting/global_reporting.htm [consulté le 28 août 2012].

108. L'avenir que nous voulons, op. cit., $\$ 161$.

109. Lors de la $3^{\mathrm{e}}$ réunion mondiale des juristes et des associations de droit de l'environnement, tenue à Limoges les 29-30 septembre et $1{ }^{\text {er }}$ octobre 2011, afin de présenter des propositions au secrétariat de l'ONU dans le cadre de la préparation de Rio +20 , Jean-Pierre Beurier a présenté une communication intitulée «Pour une convention sur la pollution d'origine tellurique des mers et océans».

110. L'avenir que nous voulons, op. cit., $\$ 163$.

111. Ibid., \$119-124.

112. La Commission centrale pour la navigation du Rhin a été la première organisation intergouvernementale créée (1815); une autre pour le Danube est apparue peu après. Aujourd'hui de telles organisations existent pour de nombreux fleuves traversant plusieurs pays : Autorité du bassin du Niger, Commission du cours d'eau du Zambèze, Commission du Mékong... Voir Laurence Boisson de Chazournes et Mara Tignino, "Gestion internationale de l'eau et développements du droit international », dans Philippe Le Prestre (dir.), Vingt Ans après : Rio et l'avant-goût de l'avenir, Québec, Presses de l'université Laval, 2011, p. 258-259. 
par le protocole de Londres de 1999 sur l'eau et la santé, ne concerne que l'Amérique du Nord et l'Europe; la convention de New York du 21 mai 1997 (qui reprend «les règles d'Helsinki») sur le droit relatif aux utilisations des cours d'eaux internationaux à des fins autres que la navigation ${ }^{113}$ n'est toujours pas entrée en vigueur et les nappes phréatiques sont tout simplement ignorées. Le seul texte à vocation universel en la matière demeure la convention de l'UNESCO relative aux zones humides d'importance internationale ${ }^{114}$ adoptée à Ramsar en 1971. Il est vrai qu'il existe des accords entre États riverains des mêmes cours d'eau, mais le corpus juridique n'est pas adapté aux enjeux par manque de volonté politique.

La situation est pourtant préoccupante: «Sous l'effet de la pression démographique et de la contamination (principalement de la pollution chimique due à l'agriculture), les ressources mondiales en eau douce ont fortement diminué, tombant de $17000 \mathrm{~m}^{3}$ par tête en 1950 à $7300 \mathrm{~m}^{3}$ en 1995. Les écosystèmes d'eau douce ont été considérablement altérés par la surexploitation, la pollution, la dégradation des habitats et parfois à l'introduction d'espèces étrangères qui ont conduit à l'extinction ou au déclin d'environ $20 \%$ des espèces de poissons ${ }^{115}$.

Les habitants des pays les plus pauvres, notamment en Afrique subsaharienne, n'ont pas accès à une eau potable ou à une «source améliorée $^{116}$ ». On estime à près de trois milliards les personnes ne disposant pas d'un robinet d'eau chez elles ou à proximité et ne disposant pas d'assainissement de base. Cela entraîne évidemment des problèmes de santé publique, le manque d'hygiène facilitant la propagation de maladies infectieuses, certaines mortelles comme le choléra. Parmi les objectifs du millénaire adoptés par l'ONU, la réduction de moitié du nombre de personnes sans assainissement de base d'ici 2015 ne semble malheureusement guère réaliste.

113. Son article $2 a$ définit le cours d'eau international comme « un système d'eaux de surfaces et d'eaux souterraines constituant, du fait de leurs relations physiques, un ensemble unitaire et aboutissant normalement à un point d'arrivée commun ».

114. L'article $1^{\text {er }}$ de la convention les définit ainsi: «Étendues de marais, de fagnes, de tourbières, ou d'eaux naturelles ou artificielles, permanentes ou temporaires, où l'eau est statique ou courante, douce, saumâtre ou salée, y compris des étendues d'eaux marines dont la profondeur, à marée basse, n'excède pas six mètres. »

115. Les Risques émergents au XxI siècle, Paris, OCDE, 2003, p. 46.

116. Selon l'Organisation mondiale de la santé et le Fonds des Nations unies pour l'enfance (UNICEF), il s'agit des raccordements des habitations au réseau de distribution, des bornes fontaines, des puits forés, des puits creusés protégés, des sources protégées et des citernes d'eau de pluie. 
L'adoption le 28 juillet 2010 d'une résolution de l'Assemblée générale de l'ONU proclamant l'accès à l'eau potable comme un droit fondamental (122 États en faveur, aucun contre, 41 abstentions) relève de l'incantation politique et ne confère pas de droit opposable. Ce que d'aucuns appellent «le droit à l'eau» signifie que les États doivent faire en sorte que chacun puisse disposer d'une eau saine, à proximité et à un coût abordable; cela ne veut ni dire que l'eau doit être gratuite, ni que sa distribution ou son traitement doivent être nécessairement directement assurés par les pouvoirs publics. Les compagnies privées spécialisées (dont deux des principales sont des sociétés françaises ${ }^{117}$ ) peuvent fort bien concourir à cette mission de service public par le biais de concessions ou d'autres formes de gestion déléguée.

\subsubsection{Les déplacés environnementaux}

Parfois présentés par les médias comme des « réfugiés climatiques » ou « réfugiés écologiques », ces personnes ont quitté leur territoire d'origine à cause d'une dégradation - progressive ou subite - de l'environnement pour aller dans une autre région de leur pays, voire bien plus rarement dans un autre État. Il s'agit donc de personnes qui, dans la terminologie du droit international, sont des « déplacés internes » en cas de migration à l'intérieur du même État ou des «migrants » en cas de déplacement vers un autre pays. Le terme "réfugié » est impropre car il renvoie à la convention de Genève de 1951 sur le statut de réfugié - amendée par le protocole de New York de 1967 - laquelle définit un réfugié comme une personne s'exilant pour fuir une persécution en raison de sa race, de sa religion, de sa nationalité, de son appartenance à un groupe social ou de ses idées politiques et prévoit un système de reconnaissance du statut de réfugié sur une base individuelle, alors que la protection nécessitée par les déplacés environnementaux devrait être collective ${ }^{118}$.

117. Voir Pierre Bauby, «Les deux “majors” françaises de l'eau : une "valse à trois temps" (intégration, internationalisation, environnement)", dans Graciela SchneierMadanes (dir.), L'Eau mondialisée. La gouvernance en question, Paris, La Découverte, 2010, p. 91-107.

118. Voir Julien Bétaille, "Des "réfugiés écologiques" à la protection des "déplacés environnementaux" ", Hommes et migrations, $\mathrm{n}^{0}$ 1284, 2010, p. 146-147; plus largement, voir Michel Prieur et al., "Projet de convention relative au statut international des déplacés environnementaux ", Revue européenne du droit de l'environnement, 2008, $\mathrm{n}^{\mathrm{o}}$ 4, p. 381-393. 
Le changement climatique provoque en effet des mouvements massifs de populations ${ }^{119}$. Ainsi que le précise Philippe Boncour, directeur du dialogue international sur les migrations au sein de l'Organisation internationale pour les migrations (OIM), «sur les trente dernières années, 1,6 milliard de personnes ont été déplacées par la sécheresse seule, alors que 718 millions [...] ont été déplacés par des catastrophes naturelles »: «Ces déplacements de personnes quantitativement plus importants dans le cadre de processus graduels plutôt que suite à des événements catastrophiques vont se poursuivre ${ }^{120}$.»Certes, en tant que migrants, les déplacés environnementaux pourraient se prévaloir du paragraphe 157 de la déclaration, néanmoins la spécificité de leur situation milite pour un traitement spécifique, notamment afin de pouvoir traiter des afflux massifs de personnes pratiquement sans espoir de retour sur leur terre d'origine ; c'est pourquoi, la déclaration des juristes de l'environnement demande « instamment aux organes compétents des Nations unies d'entamer immédiatement des négociations en vue de donner un statut juridique international aux déplacés environnementaux ${ }^{121}{ }$.

Ces trois questions ne sont pas les seules omissions ou sujets insuffisamment traités par la conférence, à telle enseigne que la déclaration des juristes de l'environnement se prononce aussi pour l'adoption de nouveaux traités ou protocoles internationaux portant sur «le pacte international sur l'environnement et le développement, les évaluations d'impact environnemental et social, les sols, la pollution d'origine tellurique des mers et des océans, l'exploitation pétrolière offshore, le paysage, les nanotechnologies, la protection de l'environnement en cas de conflits armés, le reporting du développement durable par les entreprises, les

119. Voir Peter Walker, Josh Glasser et Shubhada Kambli, Climate Change as a Driver of Humanitarian Crises and Response, Somerville, Feinstein International Center - Tufts University, juin 2012, p. 13-14; Haut-Commissariat des Nations unies pour les réfugiés. OIM et Norwegian Refugee Council, Climate Change, Migration and Displacement: Impacts, Vulnerability and Adaptations Notions, février 2009, disponible sur http://unfcc.int/resource/docs/2008/smsn/igo/o22.pdf [consulté le 15 octobre 2011].

120. «Le cadre normatif des organisations internationales", Hommes et migrations, $\mathrm{n}^{\mathrm{o}} 1284,2010$, p. 133; voir aussi OIM, L'État des migrations dans le monde, l'avenir des migrations. Renforcer les capacités face aux changements, 2010, disponible sur http://publications.iom.int/bookstore/free/WMR_2010_FRENCH. pdf [consulté le 15 octobre 2011].

121. Point IV (et ultime) de la déclaration sur les engagements post-Rio +20 , http: //www.cidce.org/rio/pdfl\%2oappel/Déclaration|\%2opost|\%2oRio.pdf [consulté le 15 juillet 2012]. 
énergies alternatives et durables, la biodiversité et la protection foncière des espaces naturels et ruraux ${ }^{122}$ ».

Il est facile d'objecter que les États présents à Rio + 20 ne pouvaient pas « sauver le monde » en deux jours, toutefois, il est regrettable que certains aspects mineurs du développement durable ou des questions déjà abondamment traitées aient recueilli l'attention des plénipotentiaires alors que, par exemple, le problème des déplacés environnementaux nécessite une prompte réponse. C'est en cela surtout que le sommet fut décevant. D'un point de vue plus légistique, il est regrettable que l'exemple d'il y a vingt ans n'ait pas été suivi : plutôt qu'un "pavé » de 238 paragraphes mêlant pétitions de principes et digressions techniques insuffisamment détaillées, mieux eût valu affirmer ou réaffirmer une trentaine de principes en une déclaration solennelle et adopter à côté un guide de recommandations pratiques à l'instar du Programme Action 21,

N'en déplaise aux catastrophistes pour lesquels la terre ne pouvait pas attendre, Rio +20 n'est qu'une étape et les négociations sur divers accords multilatéraux sur l'environnement se poursuivent. L'idée du développement durable n'est donc pas morte, elle mobilise encore même si elle aura, dans les années qui viennent certainement besoin d'un lifting. Malgré ses omissions et imperfections, la déclaration contient quand même nombre d'utiles leviers d'action. La comparant à un livre de cuisine à moitié rédigé où on ne trouverait ni un menu entier, ni même une recette complète mais seulement la liste de certains ingrédients ${ }^{123}$, Achim Steiner, sous-secrétaire général de l'ONU et directeur exécutif du PNUE, a déclaré au lendemain du sommet: «Le résultat de Rio +20 décevra et frustrera beaucoup de monde [...] Toutefois, si les nations, les entreprises, les villes et les collectivités peuvent se concentrer sur les résultats positifs du sommet, cela nous aidera à réaliser l'avenir que nous voulons $^{124}$.»

Professeur de relations internationales Écoles d'officiers de l'Armée de l'air (Salon-de-Provence)

122. Point II de la déclaration sur les engagements post-Rio +20 , ibid.

123. Compte rendu de la conférence de l'Institut des Nations unies sur la formation et la recherche (UNITAR) : Un nouveau contrat social?, Rio de Janeiro, 23 juin 2012, http://www.unitar.org/leading-international-thinkers-call-new-social-contract. htm [consulté le 30 août 2012].

124. «Une économie verte "inclusive” acceptée par les chefs d'État lors du Sommet de Rio + 20 », http://www.unep.org/newscentre.htm, 22 juin 2012 [consulté le 29 juin 2012]. 